correlates with disease activity (assessed by the Disease Activity Score using 28 joint counts - DAS28) and affects the response to the therapy (DAS 28 variation from first evaluation).

Methods: 87 consecutive RA patients (pts) (72 women and 15 men; aged $52.4 \pm 13.2$ years; disease duration of $10.7 \pm 8.6$ years), treated with DMARDs and/or biologics (bDMARDs), were recruited during their regular visit. The inclusion criteria were the 1987 American College of Rheumatology (ACR) or ACR/EULAR 2010 classification criteria. The pts underwent to anthropometric measures (BMI); abdominal US to assess STH and VTH and derived computing of peritoneal circumference (PC); and BIA to the indices of body composition (fat-free mass index (FFMI) and fat mass index (FMI).

Results: We observed increasing values of BMI, FMI, VTH (fig. 1) and CP with the worsening of disease activity phases, evaluated by DAS 28. In particular, pts with DAS28 $\geq 5.1$ had highest BMI $(30,9 \pm 2 ; p=0,036)$, FMI $(11,5 \pm 1,6 ; p=0,05), C P$ $(92,7 \pm 12,5 \mathrm{~cm} ; \mathrm{p}=0,035)$ and VTH $(24,8 \pm 5,8 \mathrm{~mm} ; \mathrm{p}=0,046)$ than pts in less severe disease activity. By linear regression analysis the predictor of higher DAS28 is the BMI $(p=0,028)$. As regard the drug response, the predictors of DAS 28 improvement are higher FFMI $(p=0,044)$ and lower $B M I(p=0,015)$, independently by bDMARDs or DMARDs treatment. A trend to higher FMI and US AT measures was observed in female with high disease activity, in particular in menopause pts.

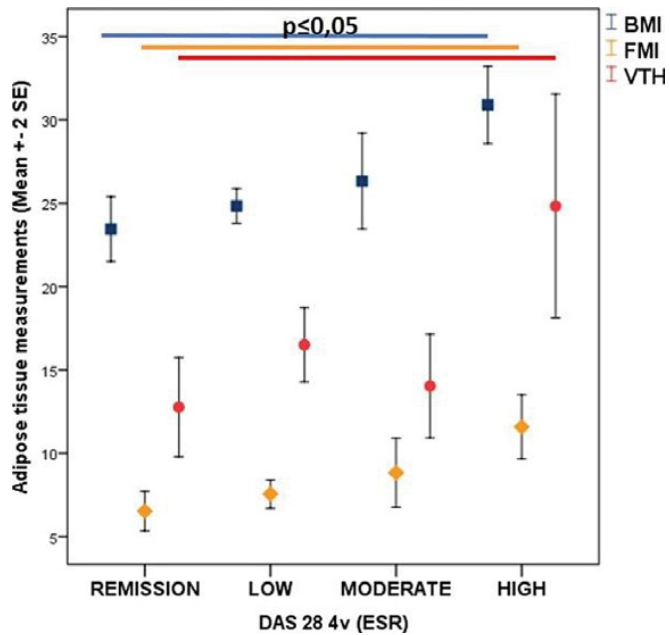

Conclusions: An altered fat distribution is observed in active RA phases; in particular, the FMI increasing is attributable just to visceral AT (VTH and CP). An inflammatory hyperactivity of visceral adiposity could be supposed in RA. The body composition, in addition to BMI, seems to predict the disease activity and drug response in RA patients. The evaluation of VTH by US could be useful to not overestimate the disease activity; instead the BIA could be a useful tool to support the clinicians in a more aggressive treatment management.

References:

[1] Nat Clin Pract Rheumatol 2007; 3(12):716-24.

[2] J Mol Endocrinol 2009; 43(1):11-8.

[3] Mediators Inflamm 2013; 2013:710928.

[4] Arthritis Care Res (Hoboken) 2012; 64(10):1471-9.

[5] Nat Rev Rheumatol 2010; 6(8):445-51.

[6] Curr Opin Clin Nutr Metab Care 2003; 6(4):387-93.

[7] Ann Rheum Dis 2007; 66(10):1316-21.

Disclosure of Interest: None declared

DOI: 10.1136/annrheumdis-2017-eular.4260

\section{AB0251 IN RHEUMATOID ARTHRITIS EROSIONS ARE MORE FREQUENT IN THE FEET THAN IN THE HANDS}

M.L. Andersson, B. Svensson, K. Forslind. Department of Clinical Sciences, Section of Rheumatology, Lund University, Lund and Helsingborg, Sweden

Background: Joint destruction in patients with rheumatoid arthritis (RA) is mostly evaluated by scoring radiographs of both hands and feet using the Sharp van der Heijde or Larsen scoring methods. In contrast, DAS28, the most commonly used composite measure of inflammation measure, does not include the feet.

Objectives: To study the distribution of erosions in hand and wrist (hands) and feet in early RA and elucidate if the feet should be included also in composite measures of inflammation

Methods: This study comprises 1052 patients from the BARFOT study of patients with early RA, recruited 1992-2006. Radiographs of hands and feet were performed at baseline, 1, 2, 5 and 8 years and evaluated by the Sharp van der Heijde scoring (SHS) method (32 joints in the hands and 12 in the feet). Disease activity at baseline was measured by the DAS28.

Results: In the feet there were significantly more eroded joints in percent of examined joints at all time points $(p<0.001)$, and higher erosion scores in percent of maximum erosion score at 5 and 8 years $(0.037$ and 0.021 respectively), compared with the hands.
There were no differences in mean joint space narrowing (JSN) between hands and feet at any time point. Patients with erosions only in the feet had significantly lower DAS28, mean 4.59, compared with the patients in the other groups, mean 5.03 in the no erosions group $\mathrm{p}=0.031$, mean 5.17 in the group with erosions only in the hands, $p=0.013$, and mean 5.15 in the group with erosions in both hands and feet, $p=0.031$.

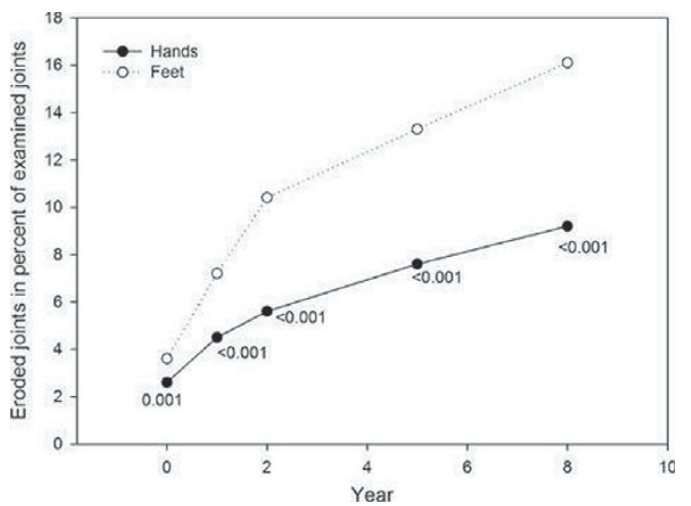

Conclusions: Joint destruction over time was more pronounced in the feet than in the hands. Baseline erosions limited to the feet were associated with low disease activity, suggesting that inflammation localized to the feet may not be reflected by DAS28. These observations may have relevance to the evaluating of disease activity and progression in the individual patient. Possibly inclusion of the feet to DAS28 might improve the validity of this disease activity measure.

Disclosure of Interest: None declared

DOI: 10.1136/annrheumdis-2017-eular.3634

\section{AB0252 THE IMPACT OF THE RHEUMATOID FOOT ON FUNCTION IN PATIENTS WITH RHEUMATOID ARTHRITIS EVALUATED BY FFI AND LFIS}

M. Erraoui, B. Amine, L. Tahiri, I. El Binoune, J. Bahha, S. Fellous, Y. Boujenane F. Allali, R. Bahiri. Rheumatology, Mohammed V University, Faculty of Medicine and Pharmacy of Rabat, El Ayachi Hospital, SALE, Morocco

Background: The impairment of function in patients with rheumatoid arthritis (RA) is determined by several factors related to the disease including joint damage. The foot, a location frequently affected during course of the disease, has a major impact on the lower limb and could cause functional disability.

Objectives: The purpose of this study is to evaluate the impact of the rheumatoid foot on function of patients with RA.

Methods: Cross-sectional study was conducted in 33 patients with RA. Patients with static lower limb disorder or foot injury from other origin were excluded. Demographic and clinical characteristics were collected: age, sex, BMI, disease duration, tender joint count, swollen joint count, foot pain evaluated on an VAS, foot squeeze test and various podiatric abnormalities observed clinically (forefoot, midfoot and rearfoot). Biological characteristics also collected: sedimentation rate in the first hour, $C$ reactive protein, rheumatoid factor and anti-CCP. Disease activity was evaluated by DAS28, CDAI, SDAI and DAS44. Functional repercussions were estimated by the French Functional Index (FFI), comprising 23 items, divided into 3 sections: pain, function and limitation of activity. Functional disability was studied by the Leeds Foot Impact Scale (LFIS), which includes 51 items (21 items specific to foot function alteration (LFIS-I) and 30 related to foot disability (LFIS-D)). Statistical analysis was performed using SPSS21 software.

Results: Thirty-three patients followed for RA were included. The mean age of our patients was $49.39 \pm 10.52$ with a female predominance $(87.9 \%)$. Mean disease duration was $9.96 \pm 7.49$ years. In all patients; 21 (95.5\%) were seropositive. The mean DAS28 was $5.53 \pm 1.38$ and the mean $\mathrm{HAQ}$ was $1.37 \pm 0.83 .93 .9 \%$ of our patients had bilateral foot pain; $69.7 \%$ in the forefoot, $18.2 \%$ in the midfoot and $42.4 \%$ in the hindfoot. The medial retromalleolar tumefaction was found in $21.2 \%$ of the patients and the lateral retromalleolar tumefaction in $45.5 \%$ of them. The squeeze test was positive in $23(69.7 \%)$ patients. Prevalences of Podiatric abnormalities were noted in the following ordre: hallux valgus $(48.5 \%)$, quintus varus $(12.1 \%)$, hallux rigidus $(6.1 \%)$, claw toe $(15.2 \%)$, triangular forefoot $(9.1 \%)$, rearfoot valgus $(27.3 \%)$ and rearfoot varus $(27.3 \%)$.

Mean FFI was $52.35 \pm 25.63$ (FFI-pain: $58.69 \pm 24.41$, FFI-function: $53.66 \pm 30.48$ and FFI- limitation of activity: $39.33 \pm 30.58$ ). Mean LFIS-I was $11.48 \pm 5.36$ and mean LFIS-D was 19.96 .

A high $\mathrm{FFI}$ was associated with foot pain $(p=0.034)$; in midfoot $(p=0.029)$ and rearfoot $(p=0.005)$ and with high disease activity: DAS28 $(P=0.005)$, CDAI, SDAI and DAS44 $(p=0.0001)$

LFIS-I and LFIS-D were statistically related to pain in foot $(\mathrm{p}=0.019)$ (midfoot $(0.042)$, hindfoot $(0.003)$ ), and to a positive squeeze test. The disease activity was positively correlated with high LFIS-I and LFIS-D $(p=0.0001)$. The presence of medial retro-malleolar swelling, posterior gutter filling and calcaneal varus is responsible for a high FFI, LFIS-I and LFIS-D with respectively: $p=0.0001$, $\mathrm{p}=0.003, \mathrm{p}=0.003$. 